\title{
Transfusion-Transmitted Malaria (TTM) in a Neonate - A Case Report
}

\section{Kailash Chand Tamaria ${ }^{1}$, Monika Matlani ${ }^{2}$ and Shyam S Meena ${ }^{1}$}

${ }^{1}$ Department of Paediatrics, VMMC and Safdarjung Hospital, New Delhi, India

${ }^{2}$ Department of Microbiology, VMMC and Safdarjung Hospital, New Delhi, India

\section{Correspondence:}

Shyam S. Meena

Department of Paediatrics

VMMC and Safdarjung Hospital,

New Delhi, India.

E-mail: dr.shyamsnmc2000@gmail.com

DOI: $10.3126 /$ jnps.v39i2.23253

Submitted on: 2019-03-23

Accepted on: 2020-03-14

Acknowledgements: None

Funding: Nil

Conflict of Interest: None declared

Permission form IRB: Yes

\section{ABSTRACT}

Transfusion-transmitted malaria in neonates is uncommon entity. Here we report a case of severe malaria in a term neonate after exchange transfusion successfully treated with intravenous artesunate.

Key words: Artesunate; neonatal malaria; transfusiontransmitted malaria (TTM); Plasmodium vivax

To cite this article: Tamaria $\mathrm{KC}$, Matlani M, Meena SS. Transfusion-Transmitted Malaria (TTM) in a Neonate - A Case report. J Nepal Paediatr Soc. 2019;39(2): $131-4$.

This work is licensed under creative common attribution 3.0 license 


\section{INTRODUCTION}

There has been a resurgence of malaria in India despite ongoing malaria control programmes. Among the unusual presentations of malaria is the occurrence of malaria in neonates. Neonates are considered relatively resistant to malaria because of the preponderance of foetal red blood corpuscles, which are relatively resistant to penetration by the malarial parasite. ${ }^{1}$ Neonatal malaria can either be due to vertical transmission from the mother following a breach in the placental barrier, or due to a fresh infection in the neonate either by transfusions or mosquito bites. ${ }^{1}$ We report a case of transfusion-transmitted Plasmodium vivax malaria in a neonate in which infection was acquired via donor blood during double volume exchange transfusion on third day of life.

\section{CASE REPORT}

A single, 40 weeks, $3.1 \mathrm{~kg}$ female baby was admitted on tenth day of life with the complaint of fever for last five days, refusal to feeds \& breathing difficulty for the past two days. On examination, the baby was febrile $\left(103^{\circ} \mathrm{F}\right)$, had pallor, icterus and respiratory distress. There was also abdominal distension with spleno-hepatomegaly. Rest of the systemic examination was normal. Initially we kept the provisional diagnosis as clinical sepsis. Sepsis work up was done and empirically antibiotics were started. After 48 hours of admission baby was still febrile $\left(103^{\circ} \mathrm{F}\right)$ with lethargy and hence the antibiotics were upgraded. On fourth day of admission, baby had also developed features of congestive cardiac failure (Tachycardia, cold periphery and prolonged capillary refilling time, increased liver size of $4 \mathrm{~cm}$ ). Sepsis workup was negative with positive $\mathrm{C}$ - reactive protein and liver functions were suggestive of hepatitis (SGOT, SGPT, ALP, S.BIL). Lumbar puncture was also within normal limits. Haemogram showed marked anaemia $(6 \mathrm{gm} / \mathrm{dl}, \mathrm{HCT} 20 \%)$ with thrombocytopenia $(45000 / \mathrm{ml})$. As the condition did not improve, detailed perinatal history was reassessed. There was history of exchange transfusion (by whole blood of known donor) on third day of life at some local hospital for neonatal hyperbilirubinemia. There was $\mathrm{Rh}$ incompatibility in the form of strong DCT positivity, jaundice and peripheral smear showing severe haemolysis. In view of severe anaemia, hepatitis and fever not responding to adequate antibiotics and history of exchange transfusion, the possibility of transfusiontransmitted infection was kept. To rule out malaria infection, baby and mother's blood were sent for rapid malaria antigen test and peripheral smear. Maternal sample was negative for malaria but baby's blood showed schizonts of P. vivax (Figure 1). Baby was started on parental artesunate in a dose schedule of $3.2 \mathrm{mg} / \mathrm{kg}$ on the first day, followed by $1.6 \mathrm{mg} / \mathrm{kg}$ for six days. The baby responded to artesunate (afebrile on eighth day of admission). The platelet counts increased gradually and the feed was gradually built up. Subsequent repeat peripheral smear examination for malarial parasites was negative. Pre exchange transfusion, the baby didn't have any hemoparasites, except for the evidence of haemolysis on peripheral smear. Mother didn't have any history of fever in the antenatal or perinatal period. So the final diagnosis was kept as transfusion-transmitted malaria. Baby was discharged on exclusive breast feeds on day 33 of life. Radical treatment was not considered because this case involved transfusion-associated malaria.

\section{DISCUSSION}

Neonatal malaria was thought to be uncommon, even in malaria endemic areas. Malarial infection in the neonate occurs predominantly through vertical transmission from the mother. Other modes of transmission including transfusion-mediated infections and acquired forms being less common. In this type of malaria, asexual forms are directly inoculated into the blood and pre-erythrocytic development of the parasite in the liver does not

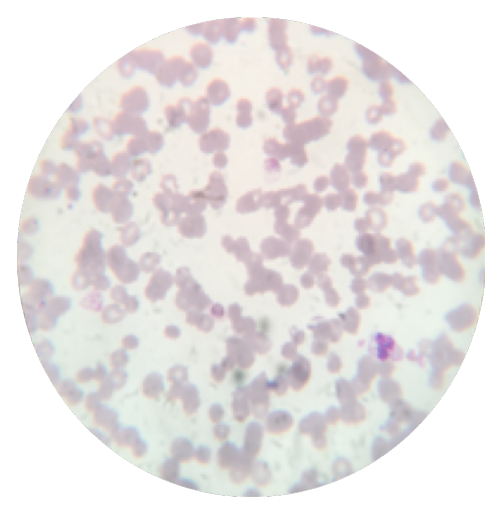

Figure 1. Peripheral smear showing multiple ring forms in red blood cells and gametocytes of Plasmodium vivax. 
occur. Transfusion malaria manifests with a shorter incubation period of two to four days as the inoculum contains the erythrocytic forms of the parasite and pre-erthrocytic phase of the life cycle within the liver does not occur. ${ }^{2}$ Unless the parasitemia is severe, malarial infection is unlikely in neonates because of presence of protective factors such as maternal antimalarial antibodies, lactoferrin, secretary IgA in human milk and $\mathrm{HbF}^{3}$

Malaria in the neonate presents as fever, refusal to feed, respiratory distress/apnea, loose motions, anaemia, jaundice, hepatosplenomegaly and thrombocytopenia. ${ }^{1}$ The symptoms and signs closely mimic sepsis and hence a high index of suspicion is needed to diagnose this condition. In our case, neonate presented with fever and had a short incubation period. There was no maternal history of fever in perinatal period as well as maternal blood for malarial parasite was also negative with history of double volume exchange transfusion on day three of life. All of these suggest that the infection in this case could be transfusion associated.

The current transfusion-transmitted malaria (TTM) occurrence rate is figured at 1 per 4 million red blood cells transfused with an estimated fatality rate of $11 \%$ in nonendemic countries as compare to endemic countries. ${ }^{5}$ TTM in the neonatal period is rare. Review of literature suggested that, in the neonatal period, malaria due to $\mathrm{P}$ vivax is more common than $\mathrm{P}$ falciparum. ${ }^{4}$ Clinically, neonatal malaria mimics bacterial sepsis. A high index of suspicion is therefore needed for making the diagnosis. Severe cases of neonatal malaria often require exchange transfusion. ${ }^{4,6}$ With the use of parenteral artesunate, we could avoid exchange transfusion in our case. This report, therefore, emphasizes the need for investigating the safety and efficacy of intravenous artesunate in the treatment of severe neonatal malaria. As per the World Health Organisation, artemisinin-based compounds (artemether, artesunate, artemether etc) are the first-line treatment of severe malaria in infants and young children, because they are superior to chloroquine in terms of schizonticidal activity, prevention of drug resistance, and early reduction of malarial transmission. ${ }^{7}$ However, there are no specific guidelines available for the treatment of severe malaria among neonates. We preferred parenteral artesunate over chloroquine and oral artesunate considering the sickness level of the neonate, the inherent risks associated with exchange transfusion, and the recent reports of chloroquine resistance among P vivax in India and the surrounding region. ${ }^{8}$

Our case report also intends to bring to the fore the unresolved issue concerning the donor screening for malaria. Current tests used to screen the donor blood, including polymerase chain reaction-based techniques, are unreliable when there is low parasitemia in the donor. ${ }^{9}$ Because of the lack of unequivocal guidelines for donor screening, TTM remains a significant problem in endemic areas. At our blood bank, we routinely screen donors by using Giemsa-stained blood smears and immunochromatographic methods detecting antigens histidine rich protein-P falciparum and parasite lactate dehydrogenase- $P$ vivax.

\section{CONCLUSIONS}

Malaria is one unusual infection in neonates and it can be acquired through blood products. It must be considered as an important differential diagnosis for suspected sepsis after exchange transfusion.

\section{REFERENCES}

1. Ibhansebhor SE. Clinical characteristics of neonatal malaria. J Trop Pediatr 1995;41:330-2. DOI: 10.1093/TROPEJ/ 41.6.330.

2. V Chauhan, RC Negi, B Verma, S Thakur. Transfusion Transmitted Malaria in a Non Endemic Area. J Association of Physicians of India 2009;57:653-654. PMID:20214005. 
3. Amaratunga C, Lopera-Mesa TM, Brittain NJ. A Role for Fetal Hemoglobin and Maternal Immune IgG in Infant Resistance to Plasmodium falciparum Malaria. Gruner AC, ed. PLoS ONE. 2011;6(4):e14798. DOI: 10.1371/ JOURNAL.PONE.0014798.

4. Thapa BR, Narang A, Bhakoo ON. Neonatal malaria. A clinical study of congenital and transfusional malaria. J Trop Pediatr 1987;33:266-8. DOI: 10.1093/TROPEJ/33.5.266.

5. Slonim AD, Bish EK, Xie RS. Red blood cell transfusion safety: probabilistic risk assessment and cost/benefits of risk reduction strategies. Ann Oper Res. 2014;221:377-406. DOI:10.1007/S10479-011-0925-0.

6. Virdi VS, Goraya JS, Khadwal A, Seth A. Neonatal transfusion malaria requiring exchange transfusion. Ann Trop Paediatr. 2003;23:205-207. DOI:10.1179/027249303322296529.

7. World Health Organization. Guidelines for the Treatment of Malaria. 2nd ed. Geneva, Switzerland: WHO Press, World Health Organization; 2010.

8. Valecha N. Recent developments in malaria chemotherapy. Proc Natl Acad Sci India. 2009;79:23-35.

9. Hänscheid T, Valadas E, Grobusch MP. Polymerase chain reaction for screening blood donors at risk for malaria: safe and useful? Emerg Infect Dis.2002;8:872-4. DOI: 10.3201/EID0808.020025. 